ANL-HEP-PR-08-31

\title{
Separating the scales of confinement and chiral-symmetry breaking in lattice QCD with fundamental quarks
}

\author{
D. K. Sinclair \\ HEP Division and Joint Theory Institute, Argonne National Laboratory, \\ 9700 South Cass Avenue, Argonne, IL 60439, USA
}

\begin{abstract}
Suggested holographic duals of QCD, based on AdS/CFT duality, predict that one should be able to vary the scales of colour confinement and chiral-symmetry breaking independently. Furthermore they suggest that such independent variation of scales can be achieved by the inclusion of extra 4-fermion interactions in QCD. We simulate lattice QCD with such extra 4-fermion terms at finite temperatures and show that for strong enough 4-fermion couplings the deconfinement transition occurs at a lower temperature than the chiral-symmetry restoration transition. Moreover the separation of these transitions depends on the size of the 4-fermion coupling, confirming the predictions from the proposed holographic dual of QCD.
\end{abstract}




\section{INTRODUCTION}

The AdS/CFT duality of Maldacena [1, 2], which indicates that $\mathcal{N}=4$ supersymmetric Yang-Mills field theory in $3+1$ dimensions is dual to $\mathcal{N}=8$ super-gravity in $4+1$ dimensions, inspired people to search for a gravity/string theory which is dual to QCD. Since such duality would map the strong-coupling regime of QCD to the weak-coupling regime of a classical gravity theory, this promises to greatly simplify certain QCD calculations. For a review of the early work in trying to construct such holographic duals for QCD without quarks, we refer the reader to the review article by Aharony [3]. The inclusion of quarks was addressed by Karch and Katz [4]. Another such model for including quark flavours was suggested by Sakai and Sugimoto [5, 6] and studied further by Antonyan, Harvey, Jensen and Kutasov [7] and Aharony, Sonnenschein and Yankielowicz [8]. These papers ([7, 8]) observe that the scales of confinement and chiral-symmetry breaking can be varied independently in these proposed QCD duals, and that the chiral-symmetry breaking scale must always be shorter than or equal to the confinement scale. Note that all these attempts at constructing holographic duals of QCD only claim validity for large $N_{c}$.

Measurement of the scales of confinement and chiral symmetry breaking can be addressed in lattice QCD by measuring the deconfinement and chiral-symmetry restoration temperatures for hot QCD. For quarks in the fundamental representation of $S U(3)$ colour, these two transitions have been observed to be coincident, within the limitations of lattice mea-

surements [9]. For this reason there have been a number of attempts to explain why this should be so [10, 11, 12, 13]. Others have argued that the scales of confinement and chiralsymmetry breaking could easily be different [14]. However, it has been observed that for quarks in the adjoint representation [15], and probably for quarks in the sextet representation of colour [16], the deconfinement temperature is significantly lower than the chiral-symmetry restoration temperature. This suggests that the real reason why these transitions appear coincident for fundamental quarks, is that the interaction between fundamental quarks and antiquarks is too weak to produce a chiral condensate at distances shorter than the confinement scale. The condensate is then produced at the confinement scale since confinement requires chiral-symmetry breaking [17, 18]. This would then make the two scales and hence the two transition temperatures identical.

The work of Antonyan, Harvey, Jensen and Kutasov [7] suggests that the introduction 
of extra 4-fermion interactions in QCD would allow the separation of the confinement and chiral-symmetry breaking scales. This makes sense, since it is known that models of the Gross-Neveu [19]/Nambu-Jona-Lasinio [20, 21] type exhibit chiral-symmetry breaking without confinement for strong enough coupling. Hence one might expect that the addition of a large enough 4-fermion coupling to QCD could produce chiral-symmetry breaking without confinement. We therefore choose to study lattice QCD with extra 4-fermion interactions of the Gross-Neveu/Nambu-Jona-Lasinio type at finite temperatures, looking for evidence for separate deconfinement and chiral-symmetry restoration transitions. Here we have been careful to make sure that the 4-fermion coupling is not so strong as to produce spontaneous chiral-symmetry breaking without gauge fields, since this phase is separated from the chiral-symmetry restored phase by a bulk transition.

Early studies of such a model showed some evidence for separate transitions for strong enough 4 -fermion interactions [22], but the lattice size $\left(8^{3} \times 4\right)$ was so small that the effect could have been a finite lattice size artifact. We study this model for large 4-fermion couplings on $16^{3} \times 4$ and $24^{3} \times 4$ lattices, and for intermediate 4-fermion couplings, on $12^{2} \times 24 \times 4,24^{3} \times 4$ and $32^{3} \times 4$ lattices (the $12^{2} \times 24 \times 4$ 'data' is from a previous study [23]). For strong 4-fermion couplings, we find that the deconfinement transition takes place at a much lower temperature than the chiral-symmetry restoration phase transition. At intermediate 4-fermion couplings, the 2 transitions are much closer, but still clearly separate. At weak 4-fermion couplings, previous work has indicated that the two transitions are coincident within the limits of our simulations [22, 23].

In section 2 we introduce the action for lattice QCD with additional 4-fermion interactions and discuss some of its properties, and how it is simulated. Section 3 presents our simulations at zero gauge coupling which are necessary to identify the limits on the 4 -fermion couplings we can use. In section 4 we describe our simulations and present results. Section 5 discusses our results, draws conclusions and indicates directions for future research.

\section{II. $\chi \mathbf{Q C D}$}

The lattice QCD action with additional 4-fermion interactions which we choose is one

we have previously called $\chi \mathrm{QCD}[22]$. This is based on the continuum Euclidean space-time 
Lagrangian density

$$
\mathcal{L}=\frac{1}{4} F_{\mu \nu} F_{\mu \nu}+\bar{\psi}(\not D+m) \psi-\frac{\lambda^{2}}{6 N_{f}}\left[(\bar{\psi} \psi)^{2}-\left(\bar{\psi} \gamma_{5} \tau_{3} \psi\right)^{2}\right] .
$$

Introducing auxiliary fields $\sigma$ and $\pi$ yields a new equivalent Lagrangian density which is quadratic in the fermion fields.

$$
\mathcal{L}=\frac{1}{4} F_{\mu \nu} F_{\mu \nu}+\bar{\psi}\left(\not D+\sigma+i \pi \gamma_{5} \tau_{3}+m\right) \psi+\frac{3 N_{f}}{2 \lambda^{2}}\left(\sigma^{2}+\pi^{2}\right)
$$

The chosen 4-fermion term is of the Gross-Neveu/Nambu-Jona-Lasinio type. It breaks flavour symmetry, but preserves the reduced chiral symmetry of the staggered-fermion implementation of quarks on the lattice. Unlike the action which includes ' $\tau_{1}$ ' and ' $\tau_{2}$ ' terms and preserves the full chiral flavour symmetry, it has a real positive fermion determinant which is essential for lattice simulations. The terms involving the chiral auxiliary fields $(\sigma$, $\pi)$ remain local in the staggered-fermion lattice transcription.

The lattice version of the action is

$$
\begin{aligned}
S= & \beta \sum_{\square}\left[1-\frac{1}{3} \operatorname{Re}\left(\operatorname{Tr}_{\square} U U U U\right)\right]+\sum_{\tilde{s}} \frac{1}{8} N_{f} \gamma\left(\sigma^{2}+\pi^{2}\right) \\
& +\sum_{f=1}^{N_{f} / 4} \sum_{s} \bar{\chi}_{f}\left[\not D+m+\frac{1}{16} \sum_{i}\left(\sigma_{i}+i \epsilon \pi_{i}\right)\right] \chi_{f}
\end{aligned}
$$

where $\not D$ is the standard staggered gauge-covariant $\not D, \epsilon=(-1)^{x+y+z+t}, s$ runs over the sites of the lattice, $\tilde{s}$ runs over the sites of the dual lattice on which the auxiliary fields reside and $i$ runs over those sites of the dual lattice adjacent to the site of the lattice on which the fermion field resides. This transcription preserves the $U(1)$ chiral symmetry of the staggered fermion formulation. $\gamma=12 / \lambda^{2}[31]$.

We simulate this action using the RHMC algorithm [24], where the fractional powers of the fermion determinant, required when the number of flavours $N_{f}$ is not a multiple of 8 , are obtained using a rational approximation to the fractional powers of the quadratic Dirac operator, and global Metropolis accept/reject steps make the algorithm exact. For subtleties associated with applying the RHMC algorithm to this action, see reference [25]. As noted in our earlier work, the presence of the 4-fermion interaction makes the Dirac operator nonsingular in the chiral $m=0$ limit. We make use of this fact to simulate at $m=0$, where there is an exact $U(1)$ chiral symmetry, so that the chiral-symmetry restoration occurs at a true phase transition at and beyond which the chiral condensate vanishes. There is, however, 
no true order parameter known for the deconfinement transition in QCD with dynamical fundamental quarks, which is seen as an abrupt jump in the Wilson Line (Polyakov Loop). Hence this transition need not necessarily be a true phase transition.

When we simulate at $m=0$, the direction in which the $U(1)$ chiral symmetry is broken is arbitrary. The chiral condensate is some linear combination of $\langle\bar{\psi} \psi\rangle$ and $i\left\langle\bar{\psi} \gamma_{5} \xi_{5} \psi\right\rangle$ or of $\sigma$ and $\pi$ ( $\xi_{5}$, the flavour version of $\gamma_{5}$ is the equivalent of $\tau_{3}$ for the 4 flavours described by a single staggered quark field). On a finite lattice, the direction defined by this chiral condensate rotates during the simulation, and the condensates average to zero since there is no spontaneous symmetry breaking in a finite volume. We therefore define an approximate order parameter, which approaches the true order parameter when the volume of the lattice becomes infinite. Experience is that $\sqrt{\bar{\psi} \psi^{2}-\bar{\psi} \gamma_{5} \xi_{5} \psi^{2}}$ or $\sqrt{\sigma^{2}+\pi^{2}}$ are good choices. Here the quantities $\bar{\psi} \psi$, etc. are lattice averages for a given gauge and auxiliary field configuration. These choices lead to some finite size rounding of the phase transition on finite lattices. From now on we shall denote these two versions of the chiral condensate as " $\langle\bar{\psi} \psi\rangle$ " and " $\langle\sigma\rangle$ ".

All our simulations have been performed with two flavours of massless quarks $\left(N_{f}=2\right)$.

\section{GROSS-NEVEU/NAMBU-JONA-LASINIO MODEL SIMULATIONS}

To determine the relevant values of $\gamma$, we perform simulations with the pure GrossNeveu/Nambu-Jona-Lasinio model, i.e. the theory described in the previous section, only without gauge fields. For these simulations, we run at zero temperature. Note that $N_{f}=2$ QCD with extra 4-fermion interactions reduces to a 6-flavour Gross-Neveu/Nambu-JonaLasinio model (2 flavours $\times 3$ colours) when the gauge coupling is set to zero. We are interested in determining the $\gamma$ value for the bulk transition at which the chiral condensate vanishes. Since, for our simulations with gauge fields, we want to determine the two finite temperature transitions, we need to simulate with 4-fermion couplings weaker than that at the bulk transition, i.e. for $\gamma>\gamma_{c}$ where $\gamma_{c}$ is $\gamma$ at the bulk chiral-symmetry restoration phase transition. In our earlier work, we simulated this theory with only 4 -fermion couplings, on an $8^{4}$ lattice. For this project we use a $12^{4}$ lattice.

We performed runs for $1.0 \leq \gamma \leq 2.5$. For each $\gamma$ we generated 500,000 length 2.5 trajectories. Figure 1 shows the chiral condensates $\langle\bar{\psi} \psi\rangle$ and $\langle\sigma\rangle$ as functions of $\gamma$ over this range. From this we estimate $\gamma_{c} \approx 1.7$. Comparing this graph with that for an $8^{4}$ lattice 
from our previous work, we observe that, as we approach $\gamma_{c}$ from below, the finite size effects only become appreciable when we get very close to the transition, as expected. The behaviour of $\langle\sigma\rangle$ can be understood from that of $\langle\bar{\psi} \psi\rangle$, since in the limit of infinite volume, these are related by

$$
\langle\bar{\psi} \psi\rangle=\gamma\langle\sigma\rangle
$$

This equation remains true when we reintroduce the gauge fields.

\section{FINITE TEMPERATURE SIMULATIONS AND RESULTS.}

We now turn to simulations with finite gauge couplings. The quark mass $m=0$ for all these simulations, so that the chiral transition is a true phase transition. Since the separation of scales of confinement and chiral-symmetry breaking is expected to occur for strong 4-fermion interactions, we start with a large 4-fermion coupling. We choose $\gamma=$ $2.5>\gamma_{c} \approx 1.7$. While this represents a large 4-fermion interaction it is not too close to the bulk transition when the gauge coupling is taken to zero. Ideally one would like to keep the gauge coupling fixed so that $\gamma$ is fixed in physical as well as lattice units, changing the temperature by changing the temporal extent (in lattice units), $N_{t}$, of the lattice. As is always the case (unless one uses anisotropic lattices) this is impractical, and we fix $N_{t}$ and vary the temperature by varying the lattice spacing in physical units by varying $\beta=6 / g^{2}$ where $g$ is the gauge coupling constant. Since we keep $\gamma$ fixed in lattice units, $\gamma$ changes in physical units as we vary $\beta$. For these simulations we use $N_{t}=4$.

Earlier simulations on $8^{3} \times 4$ lattices at $\gamma=2.5$ suggested that there could be two separate transitions. However, on such small lattices, finite size effects are large enough that definite conclusions are suspect. We have now simulated at $\gamma=2.5$ on $16^{3} \times 4$ and $24^{3} \times 4$ lattices. At each $\beta$ we ran for 50,000 length 1 trajectories, except for $\beta=5.545$, close to the deconfinement transition, where we ran for 100,000 length 1 trajectories. In figure 2 we plot the Wilson Line (Polyakov Loop), which signals the deconfinement transition and the chiral condensate, which is the order parameter for chiral-symmetry breaking, against $\beta$. The deconfinement transition, marked by a very rapid increase of the Wilson line from near-zero, occurs at a much smaller $\beta$ than the chiral phase transition, where the chiral condensate vanishes and chiral symmetry is restored. In the region of the deconfinement transition, we use Ferrenberg-Swendsen reweighting [26] to determine the position of this 


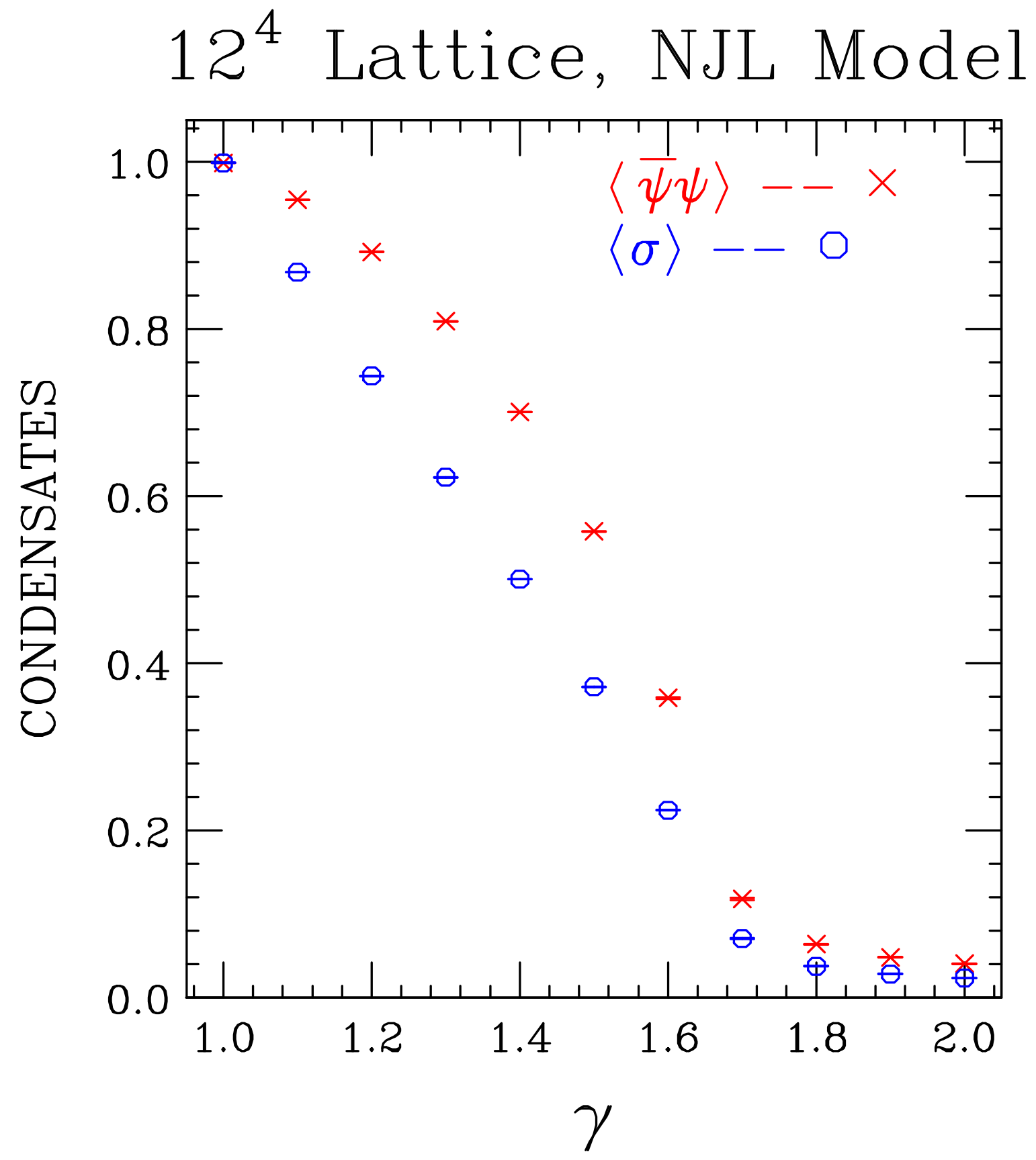

FIG. 1: The chiral condensates $\langle\bar{\psi} \psi\rangle,\langle\sigma\rangle$ for the 6-flavour Gross-Neveu/Nambu-Jona-Lasinio (zero gauge coupling) limit of QCD with extra 4-fermion couplings, as functions of $\gamma$.

transition from the Wilson line susceptibility peaks. Examining the distribution of plaquette values for each $\beta$ in the range $5.50 \leq \beta \leq 5.57$ suggests that the deconfinement $\beta, \beta_{d} \approx 5.545$. Ferrenberg-Swendsen reweighting from $\beta=5.545$ yields $\beta_{d}=5.547(3)$. The chiral transition is considerably less well measured, since there are much larger gaps between consecutive $\beta \mathrm{s}$ in its neighbourhood, and the finite size effects are large. Examining the 'data' from the two 
lattice sizes and trying to take into account the finite size effects leads us to $\beta_{\chi}=7.0(2)$ as our estimate for the $\beta$ at the chiral phase transition. Thus, for $\gamma=2.5$ the deconfinement and chiral-symmetry restoration temperatures are far apart.

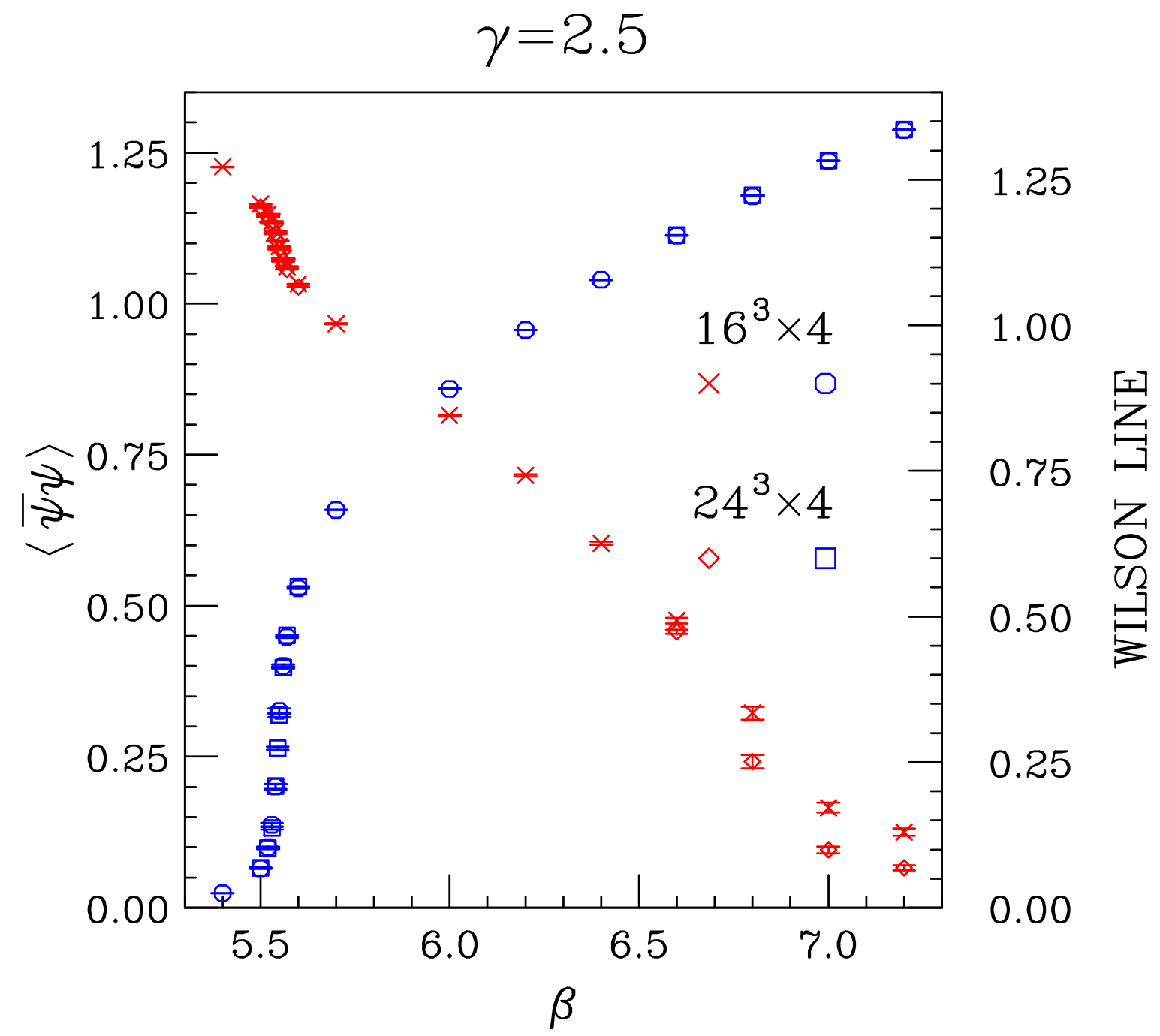

FIG. 2: Wilson line and chiral condensate as functions of $\beta$ for $\gamma=2.5$ in lattice units.

We also performed simulations at an intermediate 4-fermion coupling $\gamma=5$. Here, earlier simulations on $8^{3} \times 4$ and $12^{2} \times 24 \times 4$ lattices had failed to indicate whether there was one transition or two. We have performed simulations on $24^{3} \times 4$ lattices. To clarify chiral symmetry restoration, we also performed simulations on $32^{3} \times 4$ lattices close to the chiralsymmetry restoration transition. For the $24^{3} \times 4$ lattice we have run for 100,000 length 1 trajectories for each $\beta$ in the range $5.415 \leq \beta \leq 5.460$ and for 50,000 for those $\beta$ s outside this range. On the $32^{3} \times 4$ lattice we ran for 100,000 length 1 trajectories for each of three 
$\beta$ values. Figure 3 shows the chiral condensate and Wilson line from these new simulations along with the old results on $12^{2} \times 24 \times 4$ lattices. Again we find evidence for two separate transitions. Close to the deconfinement transition, we find that the finite size effects are very small, as was observed at $\gamma=2.5$. This is reassuring, especially in light of the fact that our old $12^{2} \times 24 \times 4$ simulations used the inexact $\mathrm{R}$ algorithm, while the new simulations used the exact RHMC algorithm. We estimate that the deconfinement $\beta, \beta_{d}=5.420(4)$. Our estimated $\beta$ for the chiral transition is $\beta_{\chi}=5.450(5)$.

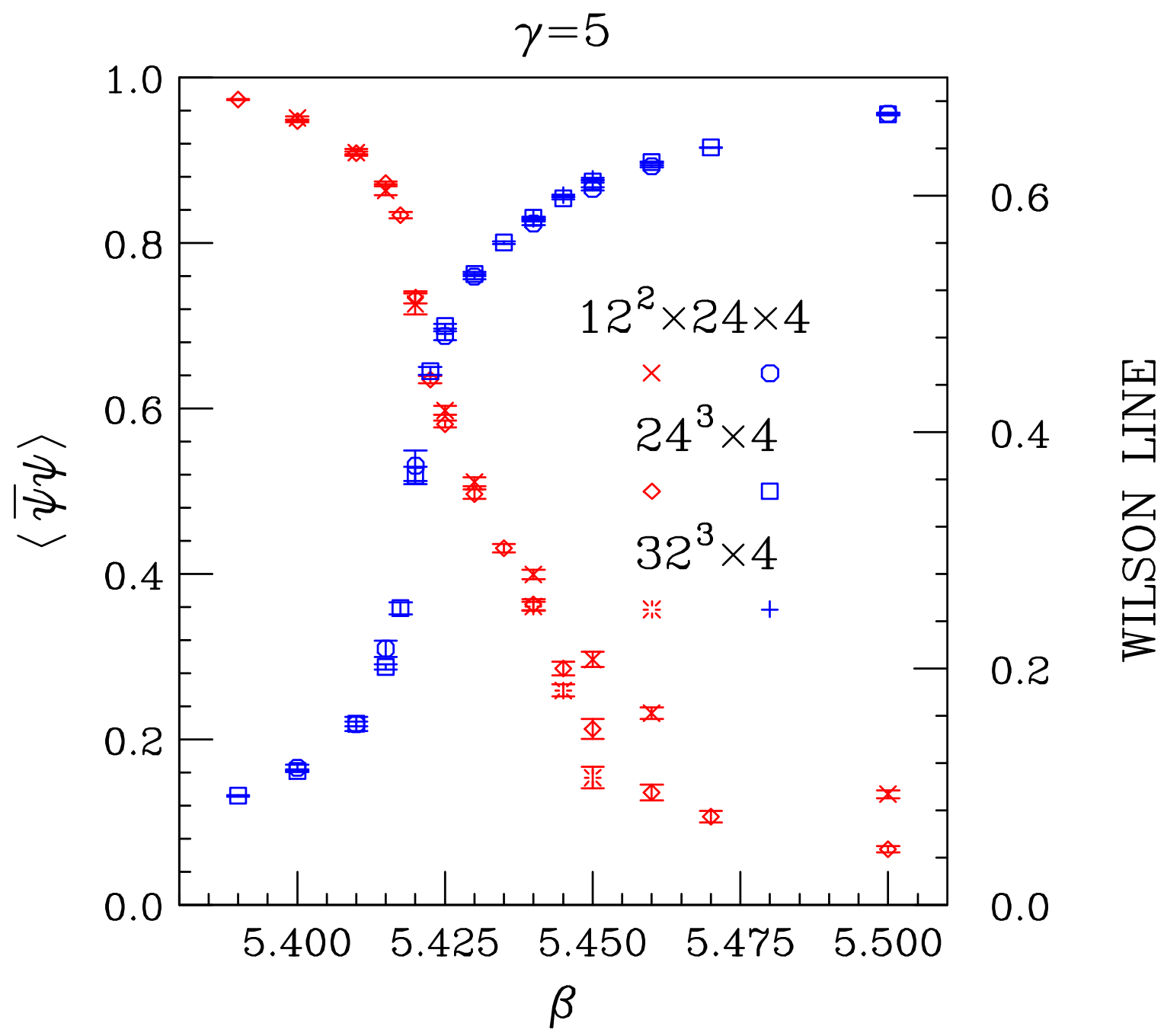

FIG. 3: Wilson line and chiral condensate as functions of $\beta$ for $\gamma=5$ in lattice units.

We know from our earlier work that if $\gamma$ is increased to $\gamma=10$, the deconfinement and chiral transitions appear to be coincident. The closeness of $\beta_{d}$ and $\beta_{\chi}$ at $\gamma=5$ compared with $\gamma=2.5$ suggests that the two transitions come together at a $\gamma$, a little above 5 . 


\section{DISCUSSION AND CONCLUSIONS}

We have simulated lattice QCD with extra 4-fermion interactions of the GrossNeveu/Nambu-Jona-Lasinio type at finite temperatures, and have observed that, for strong enough 4-fermion couplings, the deconfinement and chiral-symmetry restoration temperatures are different. Increasing the 4-fermion coupling increases the separation of these two transitions, and the deconfinement temperature is always less than or equal to the chiral-symmetry restoration temperature. This agrees with the predictions from proposed holographic duals of QCD [7, 8].

These results can be easily understood, since Gross-Neveu/Nambu-Jona-Lasinio models without gauge fields can exhibit spontaneous chiral-symmetry breaking, but do not confine the fermions. It also indicates that the reason why for QCD without such terms, the two transitions appear coincident is merely that the eigenvalue of the quadratic Casimir operator for the fundamental representation of $S U(3)_{\text {colour }}$ is too small for the QCD interactions to produce chiral-symmetry breaking at distances less than the confinement scale. Confinement then forces chiral symmetry breaking and the two scales are identical.

When the two scales are different, chiral symmetry is broken on both sides of the deconfinement transition. Hence, at this transition, the gauge fields see 'constituent quarks', i.e. quarks with dynamical masses produced by spontaneous chiral-symmetry breaking, rather than the massless 'current quarks'. These are less effective at screening colour than the 'current quarks' and the deconfinement temperature increases. We expect that in the limit of large 4-fermion couplings the dynamical quark masses will increase without bound and the deconfinement temperature will approach its quenched value. This explains why the transition coupling $\beta_{d}$ and hence temperature $T_{d}$ vary over a limited range as the 4fermion coupling is varied. These arguments suggest $5.25 \lesssim \beta_{d} \lesssim 5.6925$, where the lower limit is from simulations without the 4-fermion interaction [27, 28] and the upper limit is the quenched value [29, 30], over the whole range of $\gamma$ (the inverse 4-fermion coupling), $\gamma_{c}<\gamma<\infty$, where $\gamma_{c} \approx 1.7$ is the bulk transition $\gamma$ when the gauge couplings are switched off. On the other hand, the chiral-symmetry restoration temperature $T_{\chi}$ and coupling $\beta_{\chi}$

will vary from $T_{\chi}=T_{d}$ and $\beta_{\chi}=\beta_{d}$ for $\gamma>\gamma_{0}$ (weak 4-fermion coupling) to $\infty$ as gamma is decreased to $\gamma=\gamma_{c}$, when chiral-symmetry is always broken [32]. Our simulations indicate that $\gamma_{0}$, the $\gamma$ value for which the two transitions coalesce, lies between 5 and 10 , and is 
probably closer to 5 .

We have simulated at $\gamma=2.5$ (strong 4-fermion coupling) and $\gamma=5$ (intermediate 4fermion coupling) and previously at $\gamma=10$ and $\gamma=20$ (both weak 4-fermion couplings) [23]. The deconfinement $\beta$ s are $\beta_{d}(2.5)=5.547(3), \beta_{d}(5)=5.420(4), \beta_{d}(10)=5.327(2)$ and $\beta_{d}(20)=5.289(1)$, consistent with the above bounds, and increasing as expected with increasing 4-fermion coupling. The $\beta \mathrm{s}$ for the chiral transition are $\beta_{\chi}(2.5)=7.0(2)$, $\beta_{\chi}(5)=5.450(5), \beta_{\chi}(10)=\beta_{d}(10)$ and $\beta_{\chi}(20)=\beta_{d}(20)$. For $\gamma=2.5$ (strong 4-fermion coupling) crude estimates based on quenched running of the coupling constant, from earlier lattice simulations, indicate that $T_{\chi}$ is an order of magnitude larger than $T_{d}$, while for intermediate 4-fermion coupling, $\gamma=5$, 2-flavour 2-loop running of the coupling constant yields $T_{\chi} \approx 1.04 T_{d}$.

In our model, the 4-fermion interaction is completely local and thus irrelevant in the renormalization-group sense. Thus it will vanish in the continuum limit when the lattice spacing goes to zero, and we will no longer have two separate transitions. On the other hand, the 4-fermion interaction implied by the proposed holographic dual to QCD is nonlocal, which softens ultra-violet divergences introducing the possibility that it might have a non-trivial continuum limit. This point needs further investigation. Even if this does not happen, and QCD with extra 4-fermion interactions is only defined with an ultra-violet regulator such as the one provided by the lattice, it is a useful model since it allows one to study confinement and chiral-symmetry breaking independently in this ultra-violet regulated (effective) theory.

We have restricted ourselves to $N_{t}=4$ for this preliminary study. A more complete study would require extending this to larger $N_{t}$. At $N_{t}=4$, we would need more $\beta$ values to accurately pinpoint the chiral phase transition and to determine its nature. Additional work would be needed to understand the deconfinement transition. The fact that the vicinity of the deconfinement transition shows little finite-size dependence, makes it likely that, if it is indeed a phase transition, then it is first-order.

A lattice analysis of the non-local Nambu-Jona-Lasinio model suggested by the proposed string/gravity dual to QCD should be considered. It is a 4-dimensional non-gauge theory which exhibits chiral-symmetry breaking but not confinement. If it has a non-trivial continuum limit, it represents a new class of 4-dimensional field theories. The next step would be to include it in the lattice QCD action, just as we have done with the local Gross- 
Neveu/Nambu-Jona-Lasinio model, or alternatively to study the 5-dimensional gauge theory with right- and left-handed quarks pinned to separate 4-dimensional branes, which produced it.

The methods of lattice gauge theory and extensions of AdS/CFT duality to QCD and similar quantum field theories can be used to complement one another in the understanding of such theories. The particular example described in this paper shows how these ideas can be applied to further the understanding of confinement and chiral-symmetry breaking in QCD.

\section{Acknowledgements}

We thank Jeffrey Harvey and David Kutasov for asking the questions which led to these studies. We also acknowledge helpful discussions with Cosmas Zachos and Alexander Velytsky. In addition, we thank John Kogut for permitting us to use results from our previous work [23]. This research was supported in part by US Department of Energy contract DEAC02-06CH11357, and in part under a Joint Theory Institute grant. The simulations were performed on NERSC's Cray XT4, Franklin. The results from the earlier work included in figure 3 were obtained using the HP Superdome at the University of Kentucky under an NRAC grant.

[1] J. M. Maldacena, Adv. Theor. Math. Phys. 2, 231 (1998) [Int. J. Theor. Phys. 38, 1113 (1999)] arXiv:hep-th/9711200].

[2] O. Aharony, S. S. Gubser, J. M. Maldacena, H. Ooguri and Y. Oz, Phys. Rept. 323, 183 (2000) arXiv:hep-th/9905111.

[3] O. Aharony, arXiv:hep-th/0212193.

[4] A. Karch and E. Katz, JHEP 0206, 043 (2002) arXiv:hep-th/0205236.

[5] T. Sakai and S. Sugimoto, Prog. Theor. Phys. 113, 843 (2005) arXiv:hep-th/0412141].

[6] T. Sakai and S. Sugimoto, Prog. Theor. Phys. 114, 1083 (2006) arXiv:hep-th/0507073.

[7] E. Antonyan, J. A. Harvey, S. Jensen and D. Kutasov, arXiv:hep-th/0604017.

[8] O. Aharony, J. Sonnenschein and S. Yankielowicz, Annals Phys. 322, 1420 (2007) 
arXiv:hep-th/0604161].

[9] J. Polonyi, H. W. Wyld, J. B. Kogut, J. Shigemitsu and D. K. Sinclair, Phys. Rev. Lett. 53, 644 (1984).

[10] S. Digal, E. Laermann and H. Satz, Eur. Phys. J. C 18, 583 (2001) arXiv:hep-ph/0007175].

[11] A. Mocsy, F. Sannino and K. Tuominen, Phys. Rev. Lett. 92, 182302 (2004) arXiv:hep-ph/0308135.

[12] K. Fukushima, Phys. Rev. D 68, 045004 (2003) arXiv:hep-ph/0303225.

[13] Y. Hatta and K. Fukushima, Phys. Rev. D 69, 097502 (2004) arXiv:hep-ph/0307068.

[14] F. Gross and J. Milana, Phys. Rev. D 45, 969 (1992).

[15] J. Engels, S. Holtmann and T. Schulze, Nucl. Phys. B 724, 357 (2005) arXiv:hep-lat/0505008.

[16] J. B. Kogut, J. Shigemitsu and D. K. Sinclair, Phys. Lett. B 145, 239 (1984).

[17] T. Banks and A. Casher, Nucl. Phys. B 169, 103 (1980).

[18] H. Leutwyler and A. Smilga, Phys. Rev. D 46, 5607 (1992).

[19] D. J. Gross and A. Neveu, Phys. Rev. D 10, 3235 (1974).

[20] Y. Nambu and G. Jona-Lasinio, Phys. Rev. 122, 345 (1961).

[21] Y. Nambu and G. Jona-Lasinio, Phys. Rev. 124, 246 (1961).

[22] J. B. Kogut, J. F. Lagaë and D. K. Sinclair, Phys. Rev. D 58, 034504 (1998) arXiv:hep-lat/9801019].

[23] J. B. Kogut and D. K. Sinclair, arXiv:hep-lat/0211008.

[24] M. A. Clark and A. D. Kennedy, Phys. Rev. D 75, 011502 (2007) arXiv:hep-lat/0610047.

[25] J. B. Kogut and D. K. Sinclair, Phys. Rev. D 74, 114505 (2006) arXiv:hep-lat/0608017].

[26] A. M. Ferrenberg and R. H. Swendsen, Phys. Rev. Lett. 61, 2635 (1988).

[27] C. W. Bernard et al., Phys. Rev. D 61, 054503 (2000) arXiv:hep-lat/9908008.

[28] M. D'Elia, A. Di Giacomo and C. Pica, Phys. Rev. D 72, 114510 (2005) [arXiv:hep-lat/0503030].

[29] F. R. Brown, N. H. Christ, Y. F. Deng, M. S. Gao and T. J. Woch, Phys. Rev. Lett. 61, 2058 (1988).

[30] P. Bacilieri et al., Phys. Rev. Lett. 61, 1545 (1988).

[31] The factor of 4 difference between this and the identification in earlier papers is because there we followed earlier work on the Nambu-Jona-Lasinio model, where $N_{f}$ was the number of staggered fermion fields rather than the number of (continuum) flavours. 
[32] Strictly speaking this occurs at $\gamma=\gamma_{c}(T)<\gamma_{c}(0)$ because, at $N_{t}=4$, the pure 4 -fermion theory is also at a finite temperature $T$. 